\title{
Tadalafil para o Tratamento da Hipertensão Arterial Pulmonar Idiopática
}

\author{
Tadalafil as Treatment for Idiopathic Pulmonary Arterial Hypertension \\ Adriana Castro de Carvalho, André Luiz Hovnanian, Caio Julio César dos Santos Fernandes, Mônica Lapa, Carlos \\ Jardim, Rogério Souza \\ Grupo de Hipertensão Pulmonar - Disciplina de Pneumologia - Instituto do Coração do Hospital das Clínicas - FMUSP - São Paulo, SP
}

O uso de inibidores de fosfodiesterase, mais especificamente o sildenafil, no tratamento da hipertensão arterial pulmonar mostrou bons resultados, indicados por melhora dos parâmetros hemodinâmicos e da capacidade funcional. Poucos estudos existem a respeito dos efeitos de seus análogos como o tadalafil. O presente caso refere-se a uma paciente com hipertensão arterial pulmonar idiopática em classe funcional IV (NYHA) com resposta significativa ao uso de tadalafil.
Phosphodiesterase inhibitors like sildenafil have already been shown to improve functional capacity and hemodynamics in the treatment of pulmonary arterial hypertension. Few studies address the effects of new phosphodiesterase inhibitors as tadalafil. We report a case of a patient with idiopathic pulmonary arterial hypertension in functional class IV (New York Heart Association) with significant response to treatment with tadalafil.
A hipertensão arterial pulmonar (HAP) é uma doença caracterizada pela proliferação e remodelamento vascular que resulta no aumento progressivo da resistência vascular pulmonar com conseqüente disfunção ventricular direita e finalmente óbito ${ }^{1}$. O diagnóstico da HAP idiopática é feito quando são excluídos outros fatores causais².

O tratamento da HAP tem avançado nos últimos anos. Infelizmente as alternativas terapêuticas disponíveis ainda são de eficácia limitada, associadas a complicações e de custo elevado ${ }^{3}$. O uso do inibidor da 5-fosfodiesterase (5PD) sildenafil tem mostrado bons resultados com melhora hemodinâmica e da capacidade funcional em pacientes com HAP. Entretanto, são necessárias várias administrações diárias e nas doses utilizadas apresenta um alto custo ${ }^{3-5}$. Novos inibidores da 5-PD foram aprovados para o tratamento da disfunção erétil, como o tadalafil e o vardenafil. Uma das principais características desses agentes é a maior meia vida, mas existem poucos estudos a respeito da eficácia e segurança destes agentes na circulação pulmonar em pacientes com $\mathrm{HAP}^{4-6}$. Apresentamos um caso de HAP com melhora clínica e hemodinâmica após o tratamento com tadalafil, um inibidor da 5-PD de ação prolongada.

\section{Relato do caso}

Paciente feminina, branca, 37 anos, com dispnéia progressiva aos esforços há três anos, submetida a um ecocardiograma no início de 2003 em outro serviço que evidenciou uma comunicação interatrial (CIA) e pressão sistólica do ventrículo direito (PSVD) estimada de $47 \mathrm{mmHg}$. A paciente foi submetida na época a uma cirurgia cardíaca para correção da CIA que não foi identificada no intra-operatório. No mesmo tempo cirúrgico foi submetida a uma biópsia pulmonar que revelou

\section{Palavras-chave}

Hipertensão arterial pulmonar; inibidores da fosfodiesterase; sildenafil; tadalafil. a presença de lesões plexiformes arteriolares compatíveis com HAP. A paciente tinha antecedente de etilismo e diagnóstico clínico de hepatopatia alcoólica- Child A, estável desde 1997, sem hipertensão portal. Houve piora progressiva da dispnéia até que, em setembro de 2003, a paciente encontrava-se em classe funcional IV (New York Heart Association - NYHA), com ecocardiograma evidenciando PSVD estimada em $77 \mathrm{mmHg}$. A paciente vinha fazendo uso de espironolactona $25 \mathrm{mg} /$ dia, digoxina $25 \mathrm{mg} /$ dia e omeprazol $20 \mathrm{mg} /$ dia.

Após consulta na internet por agentes que poderiam ser utilizados em hipertensão pulmonar, a paciente iniciou por conta própria o uso de Tadalafil 10 mg a cada 36 horas. A paciente procurou nosso serviço doze meses após o início do tratamento empírico. Nesse período apresentou melhora progressiva da dispnéia, evoluindo para classe funcional II (NYHA).

Foram então realizados exames para investigação efetiva da hipertensão pulmonar, entre eles: PPF negativos, função pulmonar, reumatograma e função tireoidiana normais; ultrasonografia de abdome mostrou discreta hipoecogenicidade do parênquima hepático sem sinais de hipertensão portal. A tomografia computadorizada de tórax mostrou apenas sinais sugestivos de hipertensão pulmonar (fig. 1). Novo ecocardiograma, após um ano de tratamento, evidenciou PSVD de $58 \mathrm{mmHg}$. Optamos pela suspensão da medicação por 72 horas para a realização de cateterismo cardíaco direito com teste agudo com vasodilatador, em que não foi verificada resposta significativa ao óxido nítrico (NO) (tab. 1).

Optou-se pela reintrodução do tadalafil, na mesma dose, com manutenção da classe funcional. O teste de caminhada 


\section{Relato de Caso}

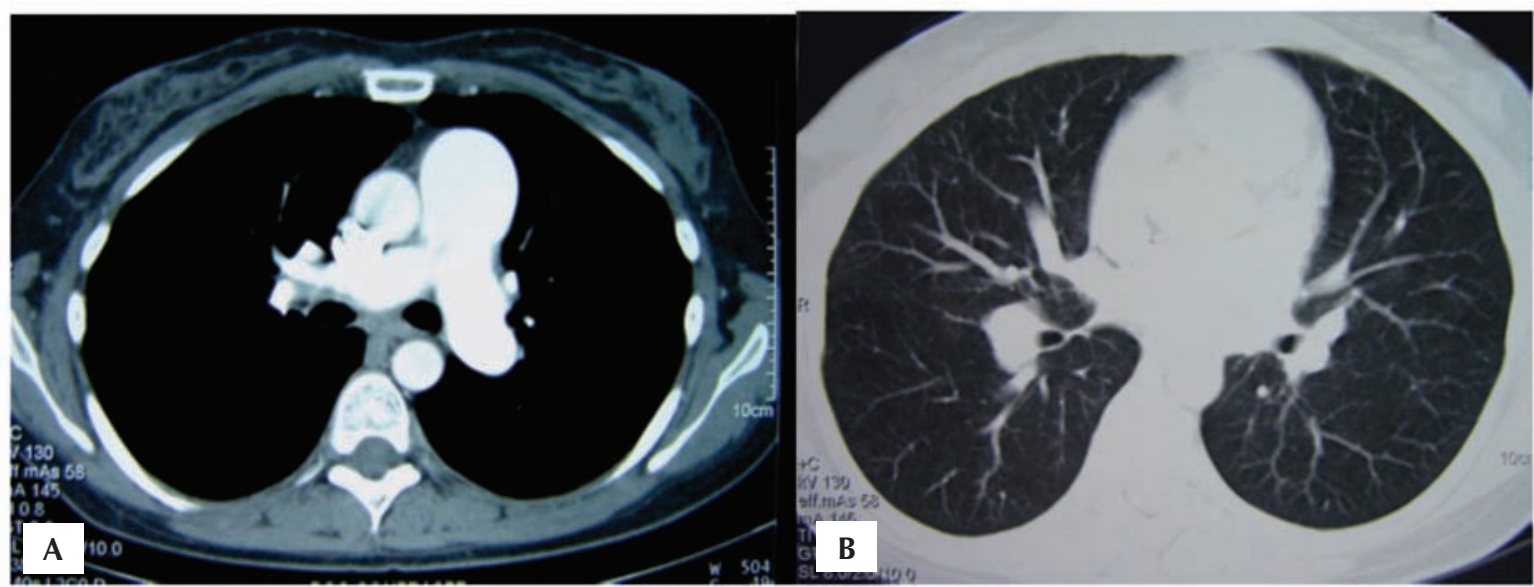

Fig. 1 - Tomografia de tórax com sinais sugestivos de hipertensão pulmonar: A) janela para mediastino ao nível do tronco da artéria pulmonar com aumento do seu diâmetro (> 2,9 cm) e aumento da relação entre os diâmetros da artéria pulmonar e da aorta (> 1); B) janela para parênquima evidenciando aumento da relação artéria/brônquio $(>1)$.

\begin{tabular}{|c|c|c|}
\hline & Basal & Pós-óxido nítrico \\
\hline $\begin{array}{l}\text { Pressão de átrio direito } \\
(\mathrm{mmHg})\end{array}$ & 5 & 5 \\
\hline $\begin{array}{l}\text { Pressão média da artéria } \\
\text { pulmonar }(\mathrm{mmHg})\end{array}$ & 40 & 34 \\
\hline $\begin{array}{l}\text { Resistência vascular } \\
\text { pulmonar (woods) }\end{array}$ & 5,27 & 4,35 \\
\hline Índice cardíaco (l/min) & 3,61 & 3,91 \\
\hline $\begin{array}{l}\text { Pressão capilar pulmonar } \\
(\mathrm{mmHg})\end{array}$ & 6 & 5 \\
\hline \multicolumn{3}{|c|}{$\begin{array}{c}\text { Tabela } 1 \text { - Dados hemodinâmicos durante o teste agudo con } \\
\text { vasodilatador }\end{array}$} \\
\hline
\end{tabular}

de seis minutos, realizado em vigência da medicação, resultou em 516 metros, partindo-se de uma freqüência cardíaca de 94 bpm, chegando a 141 bpm ao final do exame, mantendo a saturação de oxigênio pela oximetria de pulso em $93 \%$ durante todo o teste, sem suplementação de oxigênio.

\section{Discussão}

Dentre as vias fisiopatológicas mais conhecidas, três têm particular importância por serem os alvos atuais das principais alternativas terapêuticas existentes para o tratamento da HAP: a via da prostaciclina, a via da endotelina e a via do $\mathrm{NO}$. Especificamente o $\mathrm{NO}$ se apresenta como um potente vasodilatador e inibidor da proliferação muscular. O NO ativa a guanilato ciclase, que por sua vez estimula a produção de GMPc nas células musculares lisas das artérias pulmonares e promove seu relaxamento. Especificamente na HAP, os níveis de NO podem estar diminuídos ${ }^{7}$.

As fosfodiesterases são uma família de enzimas que inativam o GMPc e que possuem diferentes distribuições teciduais e afinidades ${ }^{6}$. A 5-PD está presente em grande quantidade na vasculatura pulmonar e por isso a sua inibição, que prolonga a meia-vida do GMPc e leva a uma vasodilatação, tem sido estudada como tratamento da $\mathrm{HAP}^{7}$.

O sildenafil, um inibidor seletivo da 5-PD, já mostrou ser um potente vasodilatador dose-dependente $\mathrm{e}^{3-5}$. Michelakis e cols. ${ }^{3}$ mostraram que o seu uso por três meses é seguro e está associado a melhora hemodinâmica e na capacidade funcional nos pacientes com HAP, sendo provavelmente uma alternativa mais barata aos tratamentos atuais. Já foi demonstrada melhora clínica e funcional com seu uso tanto em HAP idiopática, quanto em outras situações que cursem com hipertensão pulmonar ${ }^{8}$.

Novos inibidores seletivos da 5-PD, vardenafil e tadalafil, com o mesmo mecanismo de ação, porém com propriedades farmacocinéticas próprias foram lançados para o tratamento da disfunção erétil, mas poucos estudos existem sobre a sua utilização na HAP. Ghofrani e cols. ${ }^{6}$ compararam os efeitos hemodinâmicos do vardenafil e tadalafil com sildenafil na vasculatura pulmonar e sistêmica, em pacientes com HAP por 120 minutos. Todos os inbidores da 5-PD causaram vasodilatação pulmonar significante, mas diferiram no tempo para o seu efeito máximo e na seletividade. $\mathrm{O}$ tadalafil teve seu efeito máximo em 75 a noventa minutos (contra 40 a 45 minutos para o vardenafil e 60 minutos para o sildenafil). Tadalafil e sildenafil mostraram maior seletividade para a vasculatura pulmonar (queda no índice resistência vascular pulmonar/resistência vascular sistêmica) e apenas o sildenafil mostrou melhora na oxigenação arterial.

Palmieri e cols. ${ }^{9}$ descreveram um caso de HAP que foi tratada com tadalafil $20 \mathrm{mg}$ em dias alternados, com melhora da classe funcional de NYHA e melhora da PSVD no ecocardiograma após seis meses de tratamento. No presente caso, nossa paciente com diagnóstico de HAP, nãoresponsiva a $\mathrm{NO}$ pelo cateterismo direito, iniciou o uso do tadalafil em uma dose mais baixa $(10 \mathrm{mg})$ a cada 36 horas por conta própria após ter tido acesso a informações na mídia leiga. A paciente também apresentou melhora clínica e da classe funcional, com queda da PSVD de 77 para 58 $\mathrm{mmHg}$ no ecocardiograma, e está em uso da medicação 
há mais de um ano. Como a melhora foi evidente e não houve efeitos adversos a dose foi mantida. Além disso, o teste de caminhada da paciente encontra-se em níveis que são indicativos de bom prognóstico a longo prazo após a instituiçãa de tratamento ${ }^{10}$.

O uso dos novos inibidores da 5PD como o tadalafil podem ser uma alternativa para o tratamento da HAP e

\section{Referências}

1. Rubin LJ. Primary pulmonary hypertension. Chest. 1993; 104: 236-50.

2. Simonneau G, Galiè N, Rubin LJ, Langleben D, Seeger W, Domenighetti G, et al. Clinical classification of pulmonary hypertension. J Am Coll Cardiol. 2004; 43: 5 S- 12 S.

3. Michelakis ED, Tymchak W, Noga M, Webster L, Wu XC, Lien D, et al. Longterm treatment with oral sildenafil is safe and improves functional capacity and hemodynamics in patients with pulmonary arterial hypertension. Circulation. 2003; 108: 2066-9.

4. Wilkens H, Guth A, Konig J, Forestier N, Cremers B, Hennen B, et al. Effect of inhaled iloprost plus oral sildenafil in patients with primary pulmonary hypertension. Circulation. 2001; 104: 1218-22.

5. Michelakis ED, Tymchak W, Lien D, Webster L, Hashimoto K, Archer S. Ora sildenafil is an effective and specific pulmonary vasodilator in patients with pulmonary arterial hypertension: comparison with inhaled nitric oxide. Circulation. 2002; 105: 2398-403 podem representar uma maior aderência ao tratamento, já que a sua meia vida é maior e permite a administração única diária ou em dias alternados. São necessários ainda estudos de farmacodinâmica especificamente em pacientes com HAP a fim de se determinar a existência ou não de um componente dose-resposta e assim determinar a dose ideal de uso nessa situação.
6. Ghofrani HA, Voswinckel R, Reichenberger F, Olschewski H, Haredza P, Karadas B, et al. Differences in hemodynamic and oxygenation responses to three different phosphodiesterase-5 inhibitors in patients with pulmonary arterial hypertension. J Am Coll Cardiol. 2004; 44: 1488-96.

7. Humbert M, Sitbon O, Simonneau G. Treatment of pulmonary arterial hypertension. N Engl Med. 2004; 351(14): 1425-36.

8. Fernandes $\mathrm{CJC}$, Jardim C, Carvalho LA, Farias AQ, Terra-Filho M, Souza R. Clinical response to sildenafil in pulmonary hypertension associated to Gaucher's disease. J Inherit Metab Dis. 2005; 28(4): 603-5.

9. Palmieri EA, Lembo D, Affuso F, Fazio S. Tadalafil in primary pulmonary arterial hypertension. Ann Intern Med. 2004; 141(9): 743-4.

10. Sitbon O, Humbert M, Nunes H, Parent F, Garcia G, Herve PG, et al. Long term intravenous epoprostenol infusion in primary pulmonary hypertension: prognostic factors and survival. J Am Coll Cardiol. 2002; 40(4): 780-8. 